
SciDoc

\title{
The Effects of 6\% Hydroxyethyl Starch on Ischemia - Reperfusion Injury in Total Knee Arthroplasty with Tourniquet Application \\ Research Article
}

Jae Ho Lee ${ }^{1 *}$, Joo Huyng $\mathrm{Yoo}^{2}$, Hyun Cheol $\mathrm{Oh}^{2}$, Kyougun Park ${ }^{3}$

${ }^{1}$ Department of Anesthesiology and Pain medicine, National Health Insurance Corporation Ilsan Hospital, Ilsan, Republic of Korea.

${ }^{2}$ Department of Orthopedics, National Health Insurance Corporation Ilsan Hospital, Ilsan, Republic of Korea.

${ }^{3}$ Department of Anesthesiology and Pain Medicine, Yonsei University College of Medicine, Seoul, Republic of Korea.

\section{Abstract}

We investigated the effects of $6 \%$ hydroxyethyl starch (HES) infusion on ischemia-reperfusion injury in total knee arthroplasty using pneumatic tourniquet application. Before induction of spinal anesthesia and 15 minute after tourniquet release, we examined plasma malondialdehyde (MDA) levels to determine the degree of lipid peroxidation after ischemia-reperfusion.

Sixty patients of American Society of Anesthesiologists physical status I to II having unilateral total knee arthroplasty with tourniquet randomly assigned into 2 groups. Group NS received normal saline $10 \mathrm{ml} / \mathrm{kg}$ and group HES received hydroxyethyl starch solution $10 \mathrm{ml} / \mathrm{kg}$ respectively during spinal anesthesia. Blood samples for the analysis of plasma MDA were drawn twice at before induction and 15 minute after tourniquet release.

In group NS, plasma MDA level was not different between before and after tourniquet release $(2.20 \pm 0.36 \mathrm{nmol} / \mathrm{ml}, 2.26 \pm$ $0.43 \mathrm{nmol} / \mathrm{ml}, \mathrm{p}=0.37)$. In group HES, plasma MDA level was significantly increased before and after tourniquet $(1.84 \pm 0.43$ $\mathrm{nmol} / \mathrm{ml}, 1.96 \mathrm{nmol} / \mathrm{ml}, \mathrm{p}=0.026)$.

Hydroxyethyl starch solution have no preventive effects on lipid peroxidation after ischemic-reperfusion oxidative stress.

Keywords: 6\% Hydroxyethyl Starch; Malondialdehyde; Ischemic-Perfusion Injury; Pneumatic Tourniquet; Total Knee Arthroplasty.

\section{Introduction}

Pneumatic tourniquet is widely used in orthopedic surgery to secure a bloodless surgical field. However, this tourniquet application can cause adverse hemodynamic and metabolic reactions in patients depending on period of its application, the size of the ischemic area, the type of anesthesia administration, and the cardiovascular status of the patient [1]. Prolonged ischemia by tourniquet inflation and subsequent reperfusion by tourniquet release can cause unfavorable biochemical effects such as lipid peroxidation, that lead to tissue injury [2]. Lipid peroxidation is a chain reaction after ischemic-reperfusion of tissues that lead to oxidation of polyunsaturated fatty acids, which disrupts the structure of biological membranes and produces toxic metabolites like malondialdehyde (MDA) [3].

6\% Hydroxyethyl starch 130/0.4 (HES) is a synthetic colloid solution that can be safely used for the treatment and prevention of intravascular volume depletion. HES has a lower molecular weight and a lower degree of molar substitution but a higher C2/ C6 ratio [4].

Several recent animal studies reported that HES can reduce ischemic-reperfusion injury in which HES macromolecules reduced the microvascular permeability increased that results from ischemia $[5,6]$. Infusion of colloid solution can reduce endothelial cell activation and systemic inflammatory reaction in aortic aneurysm repair surgery [7]. In minor lower extremity surgery

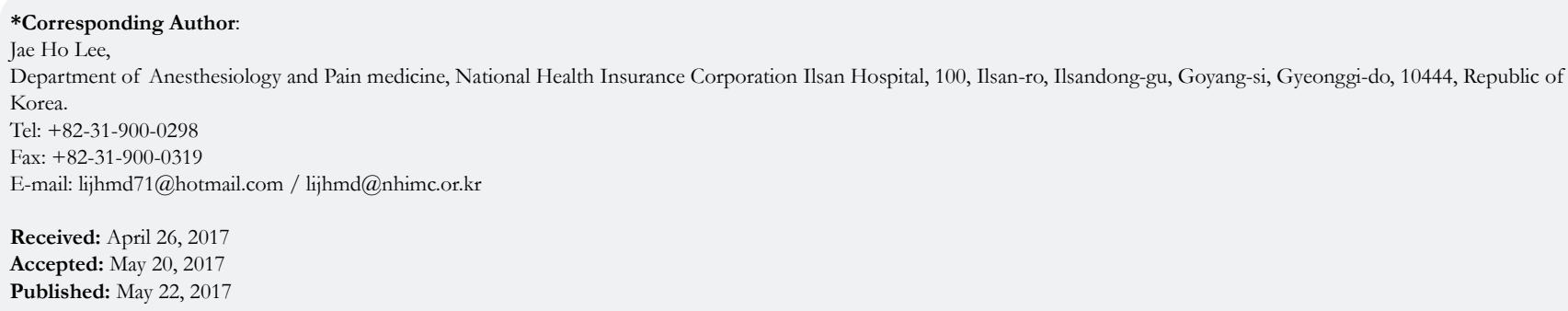

Copyright: Jae $\mathrm{Ho} \mathrm{Lee}^{\odot}$ 2017. This is an open-access article distributed under the terms of the Creative Commons Attribution License, which permits unrestricted use, distribution and reproduction in any medium, provided the original author and source are credited. 
with tourniquet application, the use of HES lowered ischemicreperfusion cell injury [8]. However, protection of tissue injury due to HES administration in major orthopedic surgery requiring long-term use of tourniquet application, such as total knee arthroplasty (TKA), is thought to be rare.

We investigated if infusion of HES would prevent tourniquet induced ischemic-reperfusion injury in TKA, by measuring plasma MDA level.

\section{Materials and Methods}

This was a single-center, prospective, randomized, single-blinded study. The study protocol was approved by the institutional review board of the National Health Insurance Corporation Ilsan Hospital (NHIMC 2016-03-044-002) and was registered with the University Hospital Medical Information Network (UMIN) Center (UMIN000022380). Written informed consent was obtained from all participants. Patients undergoing elective, primary unilateral TKA under spinal anesthesia were eligible for participation. All patients were American Society of Anesthesiologists I-II, $<80$ years of age, and gave their consent on the day before anesthesia and surgery. Exclusion criteria were absolute or relative contraindication of spinal anesthesia, use of antioxidant drug, renal impairment, electrolyte imbalance and change to general anesthesia. Patients were randomized into two groups, the normal saline (NS) group and HES group, each including 30 patients via the sealed envelope method.

On the day of surgery, each patient had 20-gauge intravenous catheter installed and were moved to operating room without premedication after a minimum 8 -hour fasting period. Before induction, each patient had an 18-gauge intravenous catheter installed in another antecubital vein and $5 \mathrm{cc}$ blood was collected for the measurement of initial MDA level (T0 BT). Heparin lock was established during the surgery. In the NS group, 0.9\% saline 10 $\mathrm{ml} / \mathrm{kg}$ was infused for 20 minutes through their 20 gauge catheter. In the HES group, HES (volulyte ${ }^{\circledR}$, Fresenius Kabi, Germany) was infused at $10 \mathrm{ml} / \mathrm{kg}$ for 20 minutes. Patients received spinalepidural combined anesthesia $\left(\right.$ Portex $^{\circledR}$, Smiths Medical, UK) with $0.5 \%$ hyperbaric bupivacaine (Bupivacaine heavy, Hana pharm, Korea) $10-12 \mathrm{mg}$ and 20 gauge epidural catheter was inserted at the L3/4 or L4/ 5 lumbar spinal level.

Systolic and diastolic blood pressures were measured every 2 minutes for the first 15 minutes via an oscillometric method after spinal anesthesia and every 5 minutes thereafter until the end of surgery. Hypotension defined as a $20 \%$ decrease of mean blood pressure and systolic blood pressure $<100 \mathrm{mmHg}$ and was treated by a bolus of 4-12 $\mathrm{mg}$ of ephedrine. Bradycardia defined as a heart rate $<50$ beats per minute and was treated with $0.2 \mathrm{mg}$ glycopyrrolate or $0.5 \mathrm{mg}$ atropine.

After confirmation that the spinal block reached above the T8 level, a pneumatic tourniquet was inflated to $320 \mathrm{mmHg}$ and TKA was started. Total infused fluid volume, hypotension, bradycardia, and tourniquet inflation time was recorded.

When surgery was completed, 15 minutes after deflation of the pneumatic tourniquet, $5 \mathrm{ml}$ of blood was withdrawn through the heparin locked 18 gauge catheter. (T15 AT) The blood samples were kept at room temperature for 30 minutesto allow clotting and were centrifuged at $3000 \mathrm{rpm}$ for 20 minutes. One milliliter of the separated serum was obtained from each sample and separately stored at $-70^{\circ} \mathrm{C}$ refrigerator.

For the determination of MDA, protein bound MDA was hydrolyzed by $6 \mathrm{M} \mathrm{NaOH}$ for 30 minutes at $60^{\circ}$. Protein was precipitated with perchloric acid and each mixture was centrifuged at $14,000 \mathrm{rpm}$ for 10 minutes. Each supernatant was transferred to an Eppendorf vial and mixed with 1,1,3,3-tetraethoxypropane(TEP; Sigma-Aldrich, USA). After incubating for 30 minutes in the dark, each reaction mixture was extracted with hexane. The upper phase was transferred to a glass tube and evaporated to dryness under a stream of nitrogen. The residue was reconstituted with mobile phase and injected into a 1200 Series, HPLC System (Agilent, USA).

We hypothesized that plasma MDA level in HES group after tourniquet deflation would be reduced by $20 \%$ relative to the control group. Given the prior report of a mean MDA level of 3.1 $\mu \mathrm{mol} / \mathrm{L}$ in the control group [8], we calculated that 30 patients per group would give a power $80 \%$ with an $\alpha$ of 0.05 with an anticipated drop-out rate of $10 \%$. The Shapiro-Wilk test was used to analyze the normal distribution of the data obtained.

All data were analyzed by SPSS 21.0 for Windows software package (SPSS, Chicago, IL, USA). Intragroup data were analyzed with paired $t$-test. Demographic data, tourniquet time and total infused volume between groups were analyzed with chi-squared test or Fisher's exact test. A p-value $<0.05$ were considered statistically significant.

\section{Results}

There were no significant differences between groups in demographic data tourniquet time and total infusion fluid use (Table 1). Mean tourniquet time was 88.3 minute all of the enrolled patients.

Two patients in the NS group and one patient in the HES group were excluded because of failure of spinal anesthesia, which necessitate a change to general anesthesia. Ten patients in the NS group and seven patients in the HES group showed hypotension. Two patients in the NS group and three patients in the HES group experienced bradycardia. Most patients were treated by ephedrine and glycopyrrolate and no patients showed any deleterious results.

In the NS group, plasma MDA level increased after tourniquet application than before tourniquet application. But it had no statistically difference. $(2.20 \pm 0.36 \mathrm{nmol} / \mathrm{ml}$ vs $2.26 \pm 0.43 \mathrm{nmol} /$ $\mathrm{ml}, \mathrm{p}=0.337)$. In the HES group, plasma MDA level increased significantly 15 minutes after tourniquet use compared with before tourniquet use $(1.84 \pm 0.43 \mathrm{nmol} / \mathrm{ml}$ vs $1.96 \pm 0.43 \mathrm{nmol} /$ $\mathrm{ml}, \mathrm{p}=0.026)$ (Table 2).

Intergroup analysis, plasma MDA in HES group was significantly lower than group NS after tourniquet release. $(2.26 \pm 0.43$ vs $1.96 \pm 0.43, \mathrm{p}<0.00)$ But, plasma MDA in the HES group was also significantly lower than group NS before tourniquet inflation $(2.20 \pm 0.36$ vs $1.84 \pm 0.43, \mathrm{p}<0.00)$. 
Table 1. Demographic Data.

\begin{tabular}{|c|c|c|c|}
\hline & $\begin{array}{c}\text { Group NS } \\
(\mathbf{n = 2 8 )}\end{array}$ & $\begin{array}{c}\text { Group HES } \\
(\mathbf{n = 2 9 )}\end{array}$ & p-value \\
\hline Gender (Male/Female) & $1 / 27$ & $1 / 28$ & \\
\hline Age(years) & $71.7 \pm 5.4$ & $71.7 \pm 5.9$ & 0.97 \\
\hline Height (cm) & $155.4 \pm 7.14$ & $155.1 \pm 5.0$ & 0.43 \\
\hline Weight (kg) & $63.4 \pm 10.2$ & $63.7 \pm 9.6$ & 0.89 \\
\hline Tourniquet time (min) & $86.4 \pm 12.3$ & $89.9 \pm 12.7$ & 0.29 \\
\hline Systolic blood pressure (mmHg) & $153.9 \pm 17.6$ & $160.1 \pm 17.6$ & 0.19 \\
\hline Diastolic blood pressure (mmHg) & $77.5 \pm 11.2$ & $79.5 \pm 11.3$ & 0.51 \\
\hline Pulse rate (BPM) & $75.7 \pm 11.5$ & $76.2 \pm 13.4$ & 0.87 \\
\hline Total amount of volume infused(ml) & $971.4 \pm 102.2$ & $945.2 \pm 203.1$ & 0.54 \\
\hline Number with hypotension & 9 & 7 & 0.41 \\
\hline Number with bradycardia & 2 & 3 & 0.67 \\
\hline
\end{tabular}

All numbers are mean \pm SD except number of case of hypotension and bradycardia.

Table 2. Comparisons of plasma MDA before tourniquet and 15 minutes after tourniquet release.

\begin{tabular}{|c|c|c|c|}
\hline & T0 BT & T15 AT & p-value \\
\hline Group NS & $2.20 \pm 0.36$ & $2.26 \pm 0.43$ & 0.34 \\
\hline Group HES & $1.84 \pm 0.43$ & $1.96 \pm 0.43$ & $0.026^{*}$ \\
\hline
\end{tabular}

All numbers are mean \pm SD. T0 BT means before tourniquet. T15 AT means 15 minute after tourniquet release. The unit is nmol/ml.

$*$ means p-value $<0.05$.

\section{Discussion}

In our study, we found that plasma MDA level slightly increased in both group after tourniquet release. In the HES group, the increase of plasma MDA level showed statistical significance $(p=0.026)$. Intergroup analysis showed that plasma MDA in HES group was significantly lower than group NS after tourniquet release. $(2.26 \pm 0.43$ vs $1.96 \pm 0.43, \mathrm{p}<0.00)$ We think this result is meaningless, because plasma MDA in the HES group was also significantly lower than group NS before tourniquet inflation. $(2.20 \pm 0.36$ vs $1.84 \pm 0.43, \mathrm{p}<0.00)$ We cannot explain why baseline plasma MDA differed between the two groups.

Interestingly, the plasma MDA level in the NS group did not increase significantly after tourniquet release, which means that lower extremity skeletal muscles are relatively resistant to ischemiareperfusion injury. In this surgery, other antioxidant were not needed preemptively.

Hypoxia produced during ischemia leads to the degradation of adenosine triphosphate (ATP) to adenosine monophosphate (AMP) and subsequently to cellular energy depletion. AMP is further degraded to adenosine or inosine monophosphate, then to inosine, and finally to xanthine and hypoxanthine [9]. When oxygen is reentered with reperfusion, hypoxanthine concentration is increased with high activity of the xanthine oxidase. This increased xanthine oxidase during ischemia leads to production of reactive oxygen species and cellular injury [10].

Lipid peroxidation is the main and most detrimental impact of free radicals on the cells. It is defined as the oxidation of multiunsaturated fatty acids by free radicals and induces structural and functional cellular damage. Lipid peroxidation leads to loss of fluidity of the membrane, reduction of membrane potential and release of cellular content. In these cascade, MDA is one of the final products of lipid peroxidation and it causes polymerization and cross-linking of membrane components, leading to altered cell surface, enzyme activity, and ionic transport. Therefore, MDA may be used as a determinant of ischemic-reperfusion cell injury, and it reaches a maximum level between 5 and 20 minutes of reperfusion $[3,11,12]$.

A number of studies have demonstrated that colloid solution may attenuate ischemic-reperfusion tissue injury. But, the precise mechanisms by which these solutions decrease tissue injury remain poorly understood. The proposed mechanism is that administration of colloid solution contributes to intravascular oncotic pressure. This augmented intravascular oncotic pressure could attenuate tissue edema and subsequent microvascular hypoperfusion during the reperfusion period. Animal studies have indicated that HES solution could work as a "sealant" at endothelial cell junctions, thereby attenuating increases of the capillary permeability after ischemia-reperfusion. The authors reported that HES molecules reduced myocardial reperfusion injury in dogs. In another study, where reperfusion injury was induced in the lower extremities of rats, it was observed that HES macromolecules reduced microvascular permeability increased by ischemia $[5,13]$. Another hypothesis is that colloid administration prevents tissue injury by augmenting plasma antioxidant potential [14]. HES has antioxidant properties and decreases neutrophil binding to endothelial cell, which could potentially decrease neutrophil-mediat- 
ed oxygen injury. It could scavenge oxidants produced by circulating xanthine oxidase, neutrophils, and other oxidants-generating systems which will prevent the oxidation of the membranes of endothelial cell.

In the case of hepatioenteric ischemia-reperfusion situation in rabbit, resuscitation with hetastach has been reported to reduce xanthine oxidase activity and preservation of plasma antioxidants [6]. In patients undergoing aortic aneurysm repair, HES infusion can reduce systemic inflammatory response and endothelial cell dysfunction associated with ischemia-reperfusion injury by evaluating microalbuminuria and vWF [7]. HES infusion was effective in lowering in ischemia-reperfusion injury caused by pneumatic tourniquet application in patients receiving minor lower extremity surgery [8].

Despite these prior observations, we did not found reduction of plasma MDA in the HES group. Both group showed an increase of plasma MDA after tourniquet release though group NS did not show statistically different. Comparing to minor lower extremity surgery [8], long term use of tourniquet application might cause more pronounced ischemic-reperfusion injury to human skeletal muscle, and HES could not prevent these injury. Another reason for this results, we might think that human skeletal muscles are more vulnerable to ischemic injury than those of animals, which diminish the protective effects to ischemia-reperfusion injury as described above.

These interpretations must be made with the awareness of the limitations of our study. If we conducted more analyses of plasma MDA level with time schedule before and after tourniquet release, it is possible that changes of plasma MDA level would have been apparent.

\section{Conclusion}

HES infusion in TKA using pneumatic tourniquet application did not prevent lipid peroxidation.

\section{References}

[1]. Girardis M, Milesi S, Donato S, Raffaelli M, Spasiano A, et al., (2000) The hemodynamic and metabolic effects of tourniquet application during knee surgery. Anesth Analg. 91(3): 727-31.

[2]. Concannon MJ, Kester CG, Welsh CF, Puckett CL (1992) Patterns of freeradical production after tourniquet ischemia: Implications for the hand surgeon. Plast Reconstr Surg. 89(5): 846-52.

[3]. Valenzuela A (1991) The biological significance of malondialdehyde determination in the assessment of tissue oxidative stress. Life Sci. 48(4): 301-9.

[4]. Gattas DJ, Dan A, Myburgh J, Billot L, Lo S, et al., (2012) Fluid resuscitation with $6 \%$ hydroxyethyl starch $(130 / 0.4)$ in acutely ill patients: An updated systematic review and meta-analysis. Anesth Analg. 114(1): 159-69.

[5]. Zikria BA, Subbarao C, Oz MC, Shih ST, McLeod PF, et al., (1989) Macromolecules reduce abnormal microvascular permeability in rat limb ischemiareperfusion injury. Crit Care Med. 17(12): 1306-9.

[6]. Nielsen VG, Tan S, Brix AE, Baird MS, Parks DA (1997) Hextend (hetastarch solution) decreases multiple organ injury and xanthine oxidase release after hepatoenteric ischemia-reperfusion in rabbits. Crit Care Med. 25(9): 1565-74.

[7]. Rittoo D, Gosling P, Simms MH, Smith SR, Vohra RK (2005) The effects of hydroxyethyl starch compared with gelofusine on activated endothelium and the systemic inflammatory response following aortic aneurysm repair. Eur J Vasc Endovasc Surg. 30(5): 520-4.

[8]. Pinar HU, Pinar A, Mavioğlu Ö, Yener N (2015) Effect of hydroxyethyl starch $130 / 0.4$ on ischemia-reperfusion determinants in minor lower extremity surgery with tourniquet application. J Clin Anesth. 27(2):105-10.

[9]. Saleem M, Ohshima H (2004) Xanthine oxidase converts nitric oxide to nitroxyl that inactivates the enzyme. Biochem Biophys Res Commun. 315(2): 455-62.

[10]. Ohshima T, Yabe Y, Ishiguro N, Iwata H (1997) Effect of dibutyryl cyclic adenosine monophosphate on skeletal muscle reperfusion injury in the rat. Eur Surg Res. 29: 438-46.

[11]. Grace PA (1994) Ischaemia-reperfusion injury. Br J Surg. 81(5): 637-47.

[12]. Girotti AW (1998) Lipid hydroperoxide generation, turnover, and effector action in biological systems. J Lipid Res. 39(8): 1529-42.

[13]. Zikria BA, Subbarao C, Oz MC, Popilkis SJ, Sachdev R, et al., (1990) Hydroxyethyl starch macromolecules reduce myocardial reperfusion injury. Arch Surg. 125(7): 930-4.

[14]. Pieper GM, Gross GJ, Kalyanaraman B (1990) An ESR study of the nitroxide radical of pentastarch-conjugated deferoxamine. Free Radic Biol Med. 9(3): 211-8 